\title{
Laboratory and field evaluation of the effects of the neonicotinoid imidacloprid on the oviposition response of Aedes (Stegomyia) aegypti Linnaeus (Diptera: Culicidae)
}

\author{
Gloria Elsa Antonio-Arreola', Roger López-Bello', Daenna Kaori Romero-Moreno', Daniel Sánchez²/+ \\ ${ }^{1}$ Instituto de Estudios Superiores de Chiapas, Escuela de Medicina Humana Campus Tapachula, Tapachula, Chiapas, México \\ ${ }^{2}$ El Colegio de la Frontera Sur, Carretera Antiguo Aeropuerto km 2.5, Tapachula, Chiapas, México
}

In this paper, we assessed the suitability of using the neonicotinoid imidacloprid with standard ovitraps by evaluating the ovicidal properties of imidacloprid and its influence on the oviposition response of gravid females of Aedes (Stegomyia) aegypti Linnaeus (Diptera: Culicidae). First, we calculated the imidacloprid lethal dose 99 (LD $(L 9)$ by exposing third instar larvae of the target species to different concentrations of the insecticide. Next, Ae. aegypti eggs were exposed to the imidacloprid $L D_{99}$ for $24 \mathrm{~h}$ and hatching inhibition was recorded. Finally, we investigated any potential repellent effect of the imidacloprid solution on the oviposition response of gravid Aedes females in field and laboratory conditions. The $L D_{99}$ obtained from larvae tests proved to be sufficient to keep any exposed eggs from hatching. No repellent effect was observed; females laid as many eggs in imidacloprid-treated ovitraps as in traps containing either clean water or temephos-treated water in both field and laboratory conditions. Our results indicate that imidacloprid is a suitable insecticide for treating ovitraps against Ae. aegypti.

Key words: Aedes aegypti - ovitrap - imidacloprid - vector control

Dengue fever is considered one of the most rapidly spreading vector-borne diseases worldwide, causing thousands of human fatalities every year despite enormous efforts aimed at reducing its prevalence (WHO 2009). It has recently re-emerged as a severe public health issue, particularly in countries located in the tropical fringe of the world. Environmental conditions in Latin America, especially the disorganised urban growth in areas with high levels of poverty, foster the breeding and dispersal of the main vector of the disease, the mosquito Aedes (Stegomyia) aegypti (Diptera: Culicidae). Vaccines and medicines against the arbovirus that causes dengue are not yet available, making vector population management the only suitable way to keep the disease from becoming an epidemic.

The reproductive biology of Ae. aegypti can be exploited to devise new technologies for controlling its population. Ovitraps are containers constructed to imitate the characteristics of Aedes' natural breeding sites to make them attractive to egg-laying females (Chadee $\&$ Corbet 1993). They are commonly used to monitor the Aedes population because: (i) one person can monitor dozens of ovitraps and (ii) they are cheap and easy to construct and little training is necessary to use them correctly (Morato et al. 2005). Moreover, with a few changes, they can be made lethal for both the adult and immature stages of Ae. aegypti (Zeichner \& Perich 1999, Chadee \& Ritchie 2010). The most straightforward modification consists in adding chemicals to the water, such

+ Corresponding author: dsanchez@ecosur.mx

Received 5 April 2011

Accepted 22 September 2011 as insecticides (Pérez et al. 2007), attractants (Chadee et al. 1993) or even a combination of both (Barbosa et al. 2007, 2010b). In this study, we use the first approach by adding the insecticide imidacloprid to standard ovitraps to convert them into lethal ovitraps.

Imidacloprid (Picus ${ }^{\circledR}$, Cheminova, Mexico) is a neonicotinoid insecticide that is more toxic to invertebrates than to vertebrates; its toxicity is due to its ability to bind nicotinic receptors in the nervous system of the animal (Anatra-Cordone \& Durkin 2005). It is widely used against crop pests (Nauen et al. 1998, Elzen 2001, Capowiez \& Berard 2006), but Paul et al. (2006) recently found that it was also effective against both the larvae and adult stages of Ae. aegypti. Moreover, Song et al. (1997) suggest that imidacloprid can be used with reasonable environmental safety and low toxicity towards non-target aquatic organisms and because it has a different mode of action than temephos, which is currently used in Mexico, cross-resistance is expected to be absent or minimal. However, to our knowledge, no study has tested the potential of using imidacloprid in ovitraps to control Ae. aegypti populations. Thus, this work focuses on evaluating the suitability of using imidacloprid in ovitraps against Ae. aegypti, paying special consideration to both the potential repellent effect of this neonicotinoid on gravid females and its ovicidal properties.

\section{MATERIALS AND METHODS}

Study site - All experiments were carried out from November 2008-March 2009 in the city of Tapachula, Chiapas, Mexico. Laboratory experiments were performed in the multidisciplinary laboratory of Escuela de Medicina of Instituto de Estudios Superiores de Chiapas Campus Tapachula. Field experiments were conducted in a local public graveyard. 
Temephos and imidacloprid - For comparison reasons, we also evaluated temephos based on the recommendation by Mexican health authorities $(1 \% \mathrm{w} / \mathrm{w}$ of a.i. in sand granules) and it was provided by the local office of vector control. Imidacloprid (Picus ${ }^{\circledR}$ ) was acquired from a local store at a $70 \% \mathrm{w} / \mathrm{w}$ a.i. powder formulation.

Imidacloprid lethal dose $99\left(L D_{99}\right)$ - Our initial objective was to determine the ovicidal properties of imidacloprid; thus, we tried to estimate the $\mathrm{LD}_{99}$ by exposing Aedes eggs to the insecticide. However, due to the high variance $(30-80 \%)$ observed in the hatching proportion in control (imidacloprid-free) groups of the Rockefeller strain maintained in the laboratory, we decided to estimate the $\mathrm{LD}_{99}$ with third instar larvae from the same strain. Six replicates consisting of six groups of 25 larvae each were exposed for $72 \mathrm{~h}$ to different concentrations $(0.000,0.005,0.010,0.050,0.100$ and $0.500 \mathrm{ppm})$ of an aqueous imidacloprid solution. In total, $750 \mathrm{Ae}$. aegypti larvae were exposed to imidacloprid and 150 were exposed to water as a control. After exposure, the larvae mortality was assessed by lightly touching the larvae with a wooden stick. Larvae that did not respond to the stimulus were considered to be dead. The $\mathrm{LD}_{99}$ estimated from these data was used in further experiments.

Repellent effect of imidacloprid - field tests - Ovitraps were constructed of black plastic containers $(5 \mathrm{~cm}$ radius, $20 \mathrm{~cm}$ height) with an ovistrip (Whatman filter paper $1,5 \mathrm{~cm} \mathrm{x} 45 \mathrm{~cm}$ ) as substrate for egg laying. Twenty replicates, each consisting of three ovitraps (1st containing $1 \mathrm{~L}$ of water, a 2 nd with $1 \mathrm{~L}$ of $1 \mathrm{ppm}$ temephos and a 3rd with $1 \mathrm{~L}$ of $0.15 \mathrm{ppm}$ imidacloprid solution, which corresponded to the $\mathrm{LD}_{99}$ estimated from the experiment described above) were deployed in the graveyard, with at least $50 \mathrm{~m}$ distance between replicates. Ovitraps within each replicate were positioned at a distance of $1 \mathrm{~m}$ from the two other ovitraps.

Each ovitrap was carefully inspected every second day for two weeks. All ovistrips were collected during each inspection regardless of the amount of eggs and were replaced with new strips. The fluid content in each ovitrap was also discarded and replaced with freshly prepared solution or plain water. Lost ovitraps were replaced when necessary. Eggs were counted from each ovistrip for the statistical analysis.

Because it was difficult to determine the species of the collected eggs, we decided to conduct similar experiments under laboratory conditions using gravid $A e$. aegypti females.

Repellent effect of imidacloprid - laboratory tests Single gravid females of the Rockefeller strain were kept in entomological cages $(30 \mathrm{~cm}$ x $50 \mathrm{~cm}$ x $50 \mathrm{~cm}$, covered with mosquito net) and offered the three solutions (water, temephos and imidacloprid) in black cups containing $250 \mathrm{~mL}$ of the solution. Eggs were collected every two days during a 10-day period.

Ovicidal properties of imidacloprid - A lethal effect is desirable when using ovitraps for vector control. Although traps are often used to monitor vector populations, lethality is required to keep ovitraps from becom- ing breeding sites. Therefore, health personnel deploy more lethal ovitraps than standard ones. To evaluate the ovicidal properties of imidacloprid, we separated 40 eggs from a single Rockefeller Aedes female in two testing groups: 20 eggs were exposed to the $\mathrm{LD}_{99}$ imidacloprid solution and 20 eggs to clean water. After $24 \mathrm{~h}$ exposure, the eggs from both treatments were moved into clean water. Hatching events were followed daily for 15 days. The total number of larvae was recorded, regardless of the time they hatched. We conducted this procedure simultaneously with the eggs from 20 females.

Statistical analyses - A probit regression was applied to mortality data to estimate the $\mathrm{LD}_{99}$ (Reyes-Villanueva et al. 1992). Any potential repellent effect was investigated by comparing the number of eggs laid in each treatment in the repellent experiments using a linear mixed model to account for time effects (repeated measures). Ovicidal properties were evaluated by applying Student's $t$ test statistics to the hatching proportion of both water and imidacloprid treatments (Antonio et al. 2009).

\section{RESULTS}

Imidacloprid $L D_{99}$ - Probit analysis estimated a $\mathrm{LD}_{99}$ of $0.15 \mathrm{ppm}$ [ $95 \%$ confidence interval $(95 \% \mathrm{CI}): 0.12-$ $0.19 \mathrm{ppm}$; model parameters: regression coefficient $=$ 3.74 , intercept $=5.37$ ] (Figure). Residuals analysis indicated a good fit between observed and estimated data $\left(\chi^{2}=2.8, \mathrm{df}=3, \mathrm{p}=0.424\right)$.

Repellent effect of imidacloprid - field tests - Ovitraps in each replicate had one of each of the following treatments: plain water, a $1.0 \mathrm{ppm}$ temephos solution or a $0.15 \mathrm{ppm}$ imidacloprid solution. A total of 11,348 eggs were collected in seven surveys in 15 days. On average, 28.66 eggs were collected per ovitrap per survey $[ \pm 1.81$, standard error (SE)]. Twenty-four ovitraps were lost and replaced. Table shows a more detailed descriptive analysis of the data. Mixed model analysis applied to the data revealed that females did not discriminate among plain

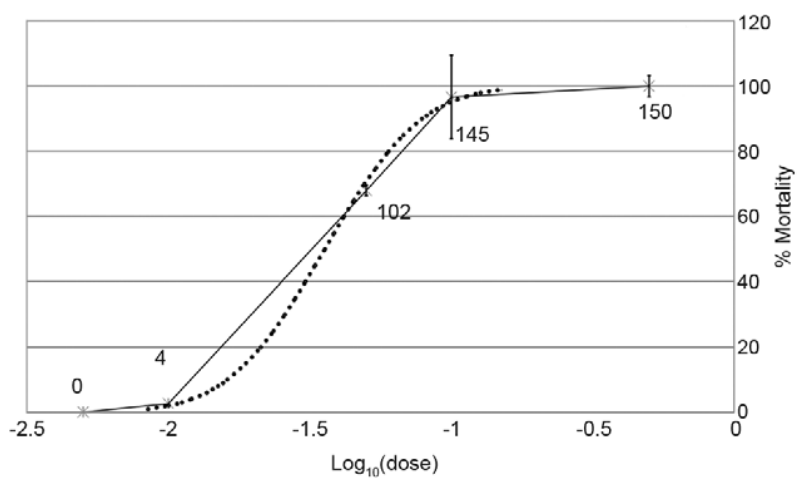

* Observed data $\cdots$ Estimated data

Curve dose-response for third instar Aedes aegypti larvae exposed to imidacloprid. Numbers along the graph are the actual number of dead larvae per concentration $( \pm 2$ standard deviation, estimated all over 6 repetitions per concentration). Control group (imidacloprid-free solution) is not shown. 
water, temephos-baited and imidacloprid-baited ovitraps $\left(\mathrm{F}_{2,1702}=0.012, \mathrm{p}=0.988\right)$; however, it detected significant differences among replicates $\left(\mathrm{F}_{19.163 .7}=4.142, \mathrm{p}<\right.$ $0.001)$ and days $\left(\mathrm{F}_{6,55.7}=16.525, \mathrm{p}<0.001\right)$. No significant interaction was found to modify the oviposition response in this experiment (treatment/replicate: $\mathrm{F}_{38}=1.076$, $\mathrm{p}=0.374$; treatment/day: $\mathrm{F}_{12,55.3}=1.32, \mathrm{p}=0.232$ ).

Repellent effect of imidacloprid - laboratory tests Ovitrap treatment solutions in this experiment were prepared at the same concentrations as in the field tests. A total of 2,875 eggs from 20 females were collected in five surveys. An average of 9.58 eggs per ovitrap per day were collected ( \pm 0.9 eggs, SE). Table shows a more detailed descriptive analysis of the data. As in the field tests, the mixed model analysis revealed that females did not discriminate between the temephos, imidacloprid and plain water solutions used in the experiment $\left(\mathrm{F}_{2,137.6}=2.210, \mathrm{p}=\right.$ $0.113)$. This analysis did not find a significant difference in the number of eggs among replicates $\left(\mathrm{F}_{19,137.6}=0.874, \mathrm{p}=\right.$ $0.615)$, but there was a significant difference in egg number among days $\left(\mathrm{F}_{4,47.6}=8.680, \mathrm{p}<0.001\right)$. No significant interaction was found to modify the oviposition response in this experiment (treatment/replicate: $\mathrm{F}_{38.88 .4}=1.056, \mathrm{p}=$ 0.407 ; treatment/day: $\mathrm{F}_{8,47.6}=0.976, \mathrm{p}=0.466$ ).

Ovicidal properties of imidacloprid - In total, 400 eggs were exposed to a $0.15 \mathrm{ppm}$ imidacloprid solution and 400 eggs to plain water (control) in the 20 replicates of the experiment. Approximately half of the eggs in the control treatment hatched $(10.7 \pm 0.95$, mean $\pm \mathrm{SE})$, while none of the imidacloprid-treated eggs hatched. Student's $t$ test showed a significant difference in the mean of both treatments (Student's $t$ test assuming different variances: $\mathrm{t}=11.275, \mathrm{df}=19, \mathrm{p}<0.001)$.

\section{DISCUSSION}

Dengue fever is a viral disease that causes thousands of human fatalities worldwide every year. Current methods to reduce its prevalence aim to suppress the populations of the mosquito vector that transmits the disease. Ovitraps are tools that take advantage of the mosquito reproductive ecology to reduce vector abundance. They have been considered to be important tools to fight $A e$. aegypti-borne diseases and are used for both monitoring (Surendran et al. 2007) and controlling (Ritchie \& Long 2003, Kittayapong et al. 2008) vector populations. In this study, we evaluated whether the addition of imidacloprid to standard ovitraps would inhibit the hatching of Ae. aegypti eggs and whether imidacloprid would cause gravid females to avoid the ovitraps.

We found that the addition of a 0.15 ppm imidacloprid solution to standard ovitraps inhibited the hatching of Ae. aegypti eggs, but did not repel egg-laying females. These results suggest that this insecticide would be useful for controlling and monitoring Ae. aegypti, as the number of eggs collected in the field and laboratory tests was not significantly different among the treatments (water-only, temephos solution and imidacloprid solution). Although we did not identify the species of the eggs collected in the field tests, the fact that the egg distribution among treatments showed a similar pattern to the laboratory results supports our conclusion. Moreover, Paul et al. (2006) found an imidacloprid $\mathrm{LD}_{50}$ of $0.084 \mathrm{ppm}$ for Ae. aegypti, more than double to the $\mathrm{LD}_{50}=0.036 \mathrm{ppm}$ found in the present study, meaning that imidacloprid or any other insecticide with a similar mode of action can be used on local Aedes populations without the concern of rapid emergence of resistance. This finding gives further support to the use of imidacloprid to fight mosquito populations in the study area.

Ovitraps treated with insecticides other than imidacloprid have yielded similar results for other species of Culicidae. Carrieri et al. (2009) found that ovitraps containing 1\% Bacillus thuringiensis var. israelensis (VectoBAC 12AS formulation) did not affect the oviposition response of gravid Aedes albopictus females and that it had a $100 \%$ lethal effect for at least 14 days, enough time to disrupt the reproductive cycle of Aedes. Methoprenetreated ovitraps also did not elicit an evasive response from gravid Ae. aegypti females, as shown in field tests in Australia by Ritchie and Long (2003). With the addition of our results with imidacloprid and temephos, there are an increasing number of methods that can be used to convert standard ovitraps into lethal ovitraps for

TABLE

Eggs collected in the field and laboratory experiments (mean \pm standard error)

\begin{tabular}{lcccccccc}
\hline \multicolumn{1}{l}{ Collection } & Collection & Collection & Collection & Collection & Collection & Collection \\
Treatment & 1 & 2 & 3 & 4 & 5 & 6 & 7 & Total \\
\hline \multicolumn{2}{l}{ Field experiments } & & & & & & & \\
$\quad$ Water & $6.53 \pm 2.011$ & $6.15 \pm 2.06$ & $40.53 \pm 7.54$ & $46.28 \pm 9.26$ & $36.65 \pm 6.96$ & $28.58 \pm 5.1$ & $38.55 \pm 10$ & $28.47 \pm 2.81$ \\
Imidacloprid & $9.74 \pm 4.52$ & $23.05 \pm 14.78$ & $21.63 \pm 6.98$ & $48.37 \pm 12.11$ & $40.75 \pm 11.84$ & $27.38 \pm 4.2$ & $27.75 \pm 5.39$ & $28.65 \pm 3.73$ \\
Temephos & $10.32 \pm 3.84$ & $11.80 \pm 2.65$ & $27.44 \pm 5.61$ & $57.17 \pm 12.57$ & $29.00 \pm 5.63$ & $27.95 \pm 4.2$ & $40.53 \pm 8.7$ & $28.84 \pm 2.81$ \\
Laboratory experiments & & & & & & & NA & $7.21 \pm 1.55$ \\
Water & $5.55 \pm 2.23$ & $3.45 \pm 2.4$ & $7.8 \pm 2.28$ & $11.15 \pm 4.63$ & $8.1 \pm 4.85$ & NA & NA & $9.97 \pm 1.54$ \\
Imidacloprid & $10.4 \pm 3.39$ & $2.6 \pm 2$ & $13.8 \pm 3.03$ & $13.65 \pm 3.63$ & $9.4 \pm 4.37$ & NA & NA \\
Temephos & $17.9 \pm 3.95$ & $1 \pm 0.69$ & $12.55 \pm 3.74$ & $16.4 \pm 3.86$ & $10 \pm 3.66$ & NA & NA & $11.57 \pm 1.61$ \\
\hline
\end{tabular}

number of eggs are pooled according to the treatment. NA: not available. 
monitoring Culicidae populations. Thus, health officers now have more options when confronted with problems of emerging insecticide resistance or when considering an integrated vector management approach.

Other studies have shown that ovitraps treated with insecticides were significantly less attractive to gravid females than insecticide-free controls (Williams et al. 2007, Coria et al. 2008). These studies reveal the importance of testing whether the addition of chemicals to ovitraps will repel insects. Nonetheless, if for any reason the use of an insecticide with repellent properties cannot be avoided, it becomes imperative to reduce the number of alternative breeding sites so that gravid Aedes females have to use the ovitraps to lay eggs (Sithiprasasna et al. 2003, Williams et al. 2007). Alternatively, attractants can be used to overcome the repellent effects of the insecticide; for instance, Culex quinquefasciatus mosquitoes are attracted to B. thuringiensis or Bacillus sphaericus-treated ovitraps if they are enhanced by adding skatole or grass infusion (Barbosa et al. 2010a) or grass infusion only (Barbosa et al. 2007), respectively.

Unexpectedly, we found that temephos-treated ovitraps were as attractive as both imidacloprid-treated and water-only ovitraps. However, given that temephos has been used for at least three decades in Mexico and that there are recent reports of temephos resistance in other countries (Rodriguez et al. 2002, Braga et al. 2004, Jirakanjanakit et al. 2007, Loke et al. 2010), its use in ovitraps should be carefully considered. Due to its different mode of action, imidacloprid can be used in areas where resistance to temephos has been documented; however, the monitoring of the resistance to both temephos and imidacloprid should be conducted simultaneously because cross-resistance cannot be completely ruled out. The rational use of imidacloprid in ovitraps could significantly reduce the possibility that the eggs of a temephos-resistant strain of Aedes will hatch, provided that the imidacloprid concentration is sufficient to kill $100 \%$ of the eggs.

Complementing research - Our results are promising, but other lines of investigation would complement the results shown here. Though some studies have found that the half-life time of imidacloprid is less than one day (see Table in Jemec et al. 2007), others describe it to be 22 days (Mailahn et al. 2008); it is thus necessary to determine the half-life time of the imidacloprid aqueous solution in field conditions using bioassays, instead of relying solely on chemical analyses, to determine how long ovitraps can be left without inspection. In addition, a large scale deployment of imidacloprid-baited ovitraps could be used to assess the potential use of imidacloprid for vector control, provided that the imidacloprid concentration remains ovicidal long enough to disrupt the life cycle of the mosquito Ae. aegypti (14 days).

\section{ACKNOWLEDGEMENTS}

To the local health authorities from Jurisdicción Sanitaria 7, that helped us during this research, and to Mr. Leonardo Sánchez Muñoz, who assisted us in both field and laboratory experiments.

\section{REFERENCES}

Anatra-Cordone M, Durkin P 2005. Imidacloprid - Human health and ecological risk assessment - Final report, USDA/Forest Service/ Forest Health Protection, New York, 283 pp.

Antonio GE, Sánchez D, Williams T, Marina CF 2009. Paradoxical effects of sublethal exposure to the naturally derived insecticide spinosad in the dengue vector mosquito, Aedes aegypti. Pest Manag Sci 65: 323-326.

Barbosa RMR, Furtado A, Regis L, Leal WS 2010a. Evaluation of an oviposition-stimulating kairomone for the yellow fever mosquito, Aedes aegypti, in Recife, Brazil. J Vector Ecol 35: 204-207.

Barbosa RMR, Regis L, Vasconcelos R, Leal WS 2010b. Culex mosquitoes (Diptera: Culicidae) egg laying in traps loaded with $\mathrm{Ba}$ cillus thuringiensis variety israelensis and baited with skatole. J Med Entomol 47: 345-348.

Barbosa RMR, Souto A, Eiras AE, Regis L 2007. Laboratory and field evaluation of an oviposition trap for Culex quinquefasciatus (Diptera: Culicidae). Mem Inst Oswaldo Cruz 102: 523-529.

Braga IA, Lima JBP, Soares SS, Valle D 2004. Aedes aegypti resistance to temephos during 2001 in several municipalities in the states of Rio de Janeiro, Sergipe and Alagoas, Brazil. Mem Inst Oswaldo Cruz 99: 199-203.

Capowiez Y, Berard A 2006. Assessment of the effects of imidacloprid on the behavior of two earthworm species (Aporrectodea nocturna and Allolobophora icterica) using 2D terraria. Ecotoxicol Environ Saf 64: 198-206.

Carrieri M, Masetti A, Albieri A, Maccagnani B, Bellini R 2009. Larvicidal activity and influence of Bacillus thuringiensis var. israelensis on Aedes albopictus oviposition in ovitraps during a two-week check interval protocol. J Am Mosq Control Assoc 25: 149-155.

Chadee DD, Corbet PS 1993. The gonotrophic status and diel pattern of entry to outdoor oviposition sites of female Aedes aegypti (L.) (Diptera: Culicidae). Ann Trop Med Parasitol 87: 263-268.

Chadee DD, Lakhan A, Ramdath WR, Persad RC 1993. Oviposition response of Aedes aegypti mosquitoes to different concentrations of hay infusion in Trinidad, West Indies. J Am Mosq Control Assoc 9: 346-348.

Chadee DD, Ritchie SA 2010. Efficacy of sticky and standard ovitraps for Aedes aegypti in Trinidad, West Indies. $J$ Vector Ecol 35: $395-400$.

Coria C, Almiron W, Valladares G, Carpinella C, Luduena F, Defago M, Palacios S 2008. Larvicide and oviposition deterrent effects of fruit and leaf extracts from Melia azedarach L. on Aedes aegypti (L.) (Diptera: Culicidae). Bioresour Technol 99: 3066-3070.

Elzen GW 2001. Lethal and sublethal effects of insecticide residues on Orius insidiosus (Hemiptera: Anthocoridae) and Geocoris punctipes (Hemiptera: Lygaeidae). J Econ Entomol 94: 55-59.

Jemec A, Tisler T, Drobne D, Sepcic K, Fournier D, Trebse P 2007. Comparative toxicity of imidacloprid, of its commercial liquid formulation and of diazinon to a non-target arthropod, the microcrustacean Daphnia magna. Chemosphere 68: 1408-1418.

Jirakanjanakit N, Saengtharatip S, Rongnoparut P, Duchon S, Bellec C, Yoksan S 2007. Trend of temephos resistance in Aedes (Stegomyia) mosquitoes in Thailand during 2003-2005. Environ Entomol 36: 506-511.

Kittayapong P, Yoksan S, Chansang U, Chansang C, Bhumiratana A 2008. Suppression of dengue transmission by application of integrated vector control strategies at sero-positive GIS-based foci. Am J Trop Med Hyg 78: 70-76.

Loke SR, Andy-Tan WA, Benjamin S, Lee HL, Sofian-Azirun M 2010. Susceptibility of field-collected Aedes aegypti (L.) (Dip- 
tera: Culicidae) to Bacillus thuringiensis israelensis and temephos. Trop Biomed 27: 493-503.

Mailahn W, Berghahn R, Meinecke S, Mohr S, Schmidt R 2008. Degradation of the insecticides Pirimicarb ${ }^{\circledR}$ and Imidacloprid ${ }^{\circledR}$ in indoor and outdoor stream mesocosms under different light conditions. Available from: umweltbundesamt.de/wasser-und-gewaesserschutz/fsa/medien/pdf/Mailahn_et_al_08_SETAC_Warschau.pdf.

Morato VC, Teixeira MG, Gomes AC, Bergamaschi DP, Barreto ML 2005. Infestation of Aedes aegypti estimated by oviposition traps in Brazil. Rev Saude Publica 39: 553-558.

Nauen R, Koob B, Elbert A 1998. Antifeedant effects of sublethal dosages of imidacloprid on Bemisia tabaci. Entomol Exp Appl 88: 287-293.

Paul A, Harrington LC, Scott JG 2006. Evaluation of novel insecticides for control of dengue vector Aedes aegypti (Diptera: Culicidae). J Med Entomol 43: 55-60.

Pérez CM, Marina CF, Bond JG, Rojas JC, Valle J, Williams T 2007. Spinosad, a naturally derived insecticide, for control of Aedes aegypti (Diptera: Culicidae): efficacy, persistence and elicited oviposition response. J Med Entomol 44: 631-638.

Reyes-Villanueva F, de la Garza-Garza H, Flores-Leal JA 1992. Effect of sublethal concentrations of abate on biological parameters of Aedes aegypti. Salud Publica Mex 34: 406-412.

Ritchie SA, Long S 2003. Does S-methoprene affect oviposition by Aedes aegypti in an ovitrap? J Am Mosq Control Assoc 19: 170-171.
Rodriguez MM, Bisset J, Ruiz M, Soca A 2002. Cross-resistance to pyrethroid and organophosphorus insecticides induced by selection with temephos in Aedes aegypti (Diptera: Culicidae) from Cuba. J Med Entomol 39: 882-888.

Sithiprasasna R, Mahapibul P, Noigamol C, Perich MJ, Zeichner BC, Burge B, Norris SL, Jones JW, Schleich SS, Coleman RE 2003. Field evaluation of a lethal ovitrap for the control of Aedes aegypti (Diptera: Culicidae) in Thailand. J Med Entomol 40: 455-462.

Song MY, Stark JD, Brown JJ 1997. Comparative toxicity of four insecticides, including imidacloprid and tebufenozide, to four aquatic arthropods. Environ Toxicol Chem 16: 2494-2500.

Surendran SN, Kajatheepan A, Sanjeefkumar KF, Jude PJ 2007. Seasonality and insecticide susceptibility of dengue vectors: an ovitrap based survey in a residential area of northern Sri Lanka. Southeast Asian J Trop Med Public Health 38: 276-282.

WHO - World Health Organization 2009. Dengue guidelines for diagnosis, treatment, prevention and control - New edition, WHO, Geneva, 147 pp.

Williams CR, Ritchie SA, Long SA, Dennison N, Russell RC 2007. Impact of a bifenthrin-treated lethal ovitrap on Aedes aegypti oviposition and mortality in north Queensland, Australia. J Med Entomol 44: 256-262.

Zeichner BC, Perich MJ 1999. Laboratory testing of a lethal ovitrap for Aedes aegypti. Med Vet Entomol 13: 234-238. 